\title{
Prevalência de tabagismo em localidade urbana da região sudeste do Brasil
}

\author{
Prevalence of smoking in a city of southeasthern Brazil \\ Cecília Amaro de Lolio*, José Maria Pacheco de Souza*, Augusto Hasiak Santo*, Cássia Maria \\ Buchalla*
}

\begin{abstract}
LOLIO, C. A. de et al. Prevalência de tabagismo em localidade urbana da região sudeste do Brasil. Rev. Saúde Pública, 27: 262-5, 1993. Foi realizado estudo transversal de prevalência da hipertensão arterial da população de 15-74 anos de idade, residente na zona urbana do Município de Araraquara, localidade situada a $250 \mathrm{~km}$ da cidade de São Paulo, Estado de São Paulo, Brasil, em 1987. Na ocasião foram perguntadas aos 1.199 entrevistados (533 do sexo masculino e 666 do sexo feminino) questōes sobre o uso de tabaco (fumo), a forma de uso, o hábito de tragar, bem como variáveis sociodemográficas. A amostra foi equiprobabilística, por conglomerados, em três estágios. A prevalência de tabagismo foi bastante alta, de $45,2 \%$ entre os homens e $22,8 \%$ entre as mulheres. Os ex-fumantes eram em percentagem de $15,9 \%$ entre os homens e $8,0 \%$ das mulheres. $O$ sexo masculino fumava maior quantidade de equivalentes de cigarro do que o feminino. As camadas de mais baixa renda familiar fumavam mais, em ambos os sexos, do que os estratos de renda mais alta. Entre os homens, a prevalência de tabagismo diminuía com a maior escolaridade e nas mulheres, este aspecto não foi notado. Comparando com os resultados já publicados sobre a alta prevalência de hipertensão arterial e de obesidade, nota-se que a população de Araraquara, cidade média do interior urbano afluente do Brasil, apresenta uma frequiência bastante alta de fatores de risco para doenças crônicas não-transmissíveis.
\end{abstract}

Descritores: Fumo, epidemiologia. Fatores de risco. Doença crônica, epidemiologia.

O tabagismo é um hábito bastante frequiente na sociedade brasileira embora mediante levantamentos pontuais, estimava-se em 1988 que $41 \%$ dos homens adultos e $37 \%$ das mulheres adultas brasileiras eram fumantes ${ }^{1}$. Esse hábito tem sido declarado importante fator de risco para a causação de inúmeras doenças, tais como, dentre outras, câncer: (pulmão, laringe, esôfago, boca, bexiga, pâncreas, rim, estômago e colo uterino), doenças ateroscleróticas (doença coronária, doença vascular periférica, derrame cerebral), doença pulmonar obstrutiva crônica e úlcera péptica ${ }^{2}$.

Araraquara é uma cidade média do Interior urbano afluente do Estado de São Paulo, com uma população estimada em 1987 de 150 mil habitantes. A primeira causa de óbito no Município é atualmente representada pelas doenças do apare-

* Departamento de Epidemiologia da Faculdade de Saúde Pública da Universidade de São Paulo - São Paulo, SP Brasil

** Fundação SEADE. São Paulo. Microfichas de óbito segundo local de residência. Dados inéditos.

Separatas/Reprints: C. A. de Lolio Av. Dr. Amaldo, 715, 01246-904-São Paulo, SP- Brasil

Edição subvencionada pela FAPESP. Processo Medicina 93/ 0208-5 lho circulatório (mais de um terço dos óbitos**). Outros estudos mostram no Município uma elevadíssima prevalência de hipertensão $\operatorname{arterial}^{3} \mathrm{e}$ também de obesidade 4 .

O objetivo do presente estudo é o de descrever a prevalência do hábito de fumar na zona urbana do Município de Araraquara, caracterizando desta forma a existência de outro fator de risco para doenças, crônicas não-transmissíveis. Tal tipo de levantamento, embora pontual, se justifica já que é impossivel obter estatísticas para o hábito de fumar para o País inteiro, mas apenas para localidades que representem adequadamente uma determinada região. Subjacente a este objetivo, está evidentemente a hipótese de que o Município de Araraquara representa uma determinada categoria de municipios do interior do Estado de São Paulo, que se convencionou designar interior urbano afluente ${ }^{5}$.

\section{Metodologia}

Em 1987, foi realizado em Araraquara estudo transversal para determinar a prevalência de hipertensão arterial na população adulta (15-74 anos), residente na zona urbana do Município ${ }^{3}$. Para tal fim, foi feita uma amostragem equiprobabilística em três etapas da população urbana do grupo etário 
de interesse, equivalente naquele ano a $68,4 \%$ da população geral ${ }^{3}$. A primeira etapa foi representada pelos setores censitários; a segunda, pelos quarteirões ou conjuntos de quarteirões; a terceira, pelos domicílios, e dentro dos domicílios, todos os indivíduos do grupo etário foram entrevistados. Os cálculos do procedimento amostral previam uma prevalência de hipertensão arterial de $17,5 \%$, o que é bastante inferior à prevalência esperada de tabagismo, motivo pelo qual resolveu-se utilizar também esses dados para o cálculo da prevalência de tabagismo. A fração de amostragem foi de 1,8\%.

Foram realizadas até três visitas para a localização dos domicílios e entrevista das pessoas. Para cada adulto (15-74 anos) residente no domicílio, inquiria-se sobre uma série de características. Dentre as variáveis de interesse para este artigo, devem ser destacadas:

- idade

- sexo

- escolaridade formal: maior grau completado

- renda familiar: em pisos nacionais de salários (PNS)

- tabagismo: definido como uso consistente de cigarros e/ou cachimbo e/ou charuto por 6 meses ou mais, todos os dias, até o momento da entrevista - ex-fumantes: entrevistados que, tendo feito uso consistente de cigarros e/ou cachimbo e/ou charuto por 6 meses ou mais, todos os dias, tenham declarado que abandonaram o hábito a qualquer tempo, no momento da entrevista

- forma de uso de tabaco: sob a forma de cigarros de papel, de palha, cachimbo, charutos (não foi computada a forma não inalatória como mascar tabaco)

- equivalentes de fumo em cigarros de papel: para os outros derivados de fumo excluindo o cigarro de papel, foi calculado um equivalente em cigarros de papel, da seguinte forma 3,5 :

cachimbo: 1 "pitada" = 2 cigarros de papel

cigarro de palha: 1 cigarro de palha $=2$ cigarros de papel

- charuto: 1 charuto $=4$ cigarros de papel

- hábito de tragar

- teor em nicotina do cigarro de papel fumado

\section{Resultados}

Foram entrevistadas 1.199 pessoas entre $15 \mathrm{e}$ 74 anos, das quais 533 homens e 666 mulheres.

A Tabela 1 mostra a prevalência de tabagismo na população de estudo, segundo idade e sexo. Nota-se que a prevalência é sempre mais alta para o sexo masculino que para o feminino e que um terço da população adulta é composto de fumantes. Mesmo na população jovem, abaixo de 24 anos, o hábito é bastante freqüente.
Tabela 1. Prevalência (\%) de tabagismo na população urbana de 15 a 74 anos segundo idade e sexo, Município de Araraquara, SP, 1987.

\begin{tabular}{lccc}
\hline \multirow{2}{*}{$\begin{array}{l}\text { Idade } \\
\text { (anos) }\end{array}$} & \multicolumn{3}{c}{ Sexo } \\
\cline { 2 - 3 } & Masculino & Feminino & Total \\
\hline $15-24$ & 32,9 & 22,9 & 27,6 \\
$25-34$ & 53,2 & 37,2 & 44,7 \\
$35-44$ & 55,5 & 18,6 & 34,0 \\
$45-54$ & 37,0 & 17,2 & 25,3 \\
$55-64$ & 44,5 & 16,5 & 28,7 \\
$65-74$ & 58,0 & 6,6 & 27,6 \\
\hline Total & 45,2 & 22,8 & 32,8 \\
\hline
\end{tabular}

A Tabela 2 apresenta a prevalência de exfumantes na população de estudo, segundo idade e sexo. Verifica-se que esta proporção é crescente com a idade: entre os homens até 55-64 anos, e entre as mulheres, até 45-54 anos. Assim como predominam os homens entre os fumantes, também os mesmos predominam entre os ex-fumantes.

Tabela 2. Prevalência (\%) de ex-fumantes na população urbana de 15 a 74 anos, segundo idade e sexo, Municipio de Araraquara, SP, 1987.

\begin{tabular}{lccr}
\hline \multirow{2}{*}{$\begin{array}{l}\text { ldade } \\
\text { (anos) }\end{array}$} & \multicolumn{3}{c}{ Sexo } \\
\cline { 2 - 3 } & Masculino & Feminino & Total \\
\hline $15-24$ & 2,9 & 4,5 & 3,5 \\
$25-34$ & 14,0 & 9,3 & 11,5 \\
$35-44$ & 13,6 & 9,7 & 11,3 \\
$45-54$ & 26,0 & 14,3 & 19,1 \\
$55-64$ & 36,9 & 4,7 & 18,7 \\
$65-74$ & 22,6 & 2,2 & 10,5 \\
\hline Total & 15,9 & 8,0 & 11,5 \\
\hline
\end{tabular}

As Tabelas 3 e 4 apresentam a prevalência de tabagismo segundo o número de cigarros de papel ou equivalência) fumados ao dia, segundo a idade, respectivamente entre os homens e as mulheres. Os homens não apenas fumam mais freqüentemente do que as mulheres, mas também consomem mais cigarros.

Ao se estudar o tipo de cigarro utilizado, 5,0\% dos homens fumavam exclusivamente cigarro de palha (todos com 45 anos e mais), um hábito típico de zona rural; e $88,8 \%$ fumavam exclusivamente cigarros de papel com filtro; $3,4 \%$ fumavam exclusivamente cigarros de papel sem filtro, e $2,5 \%$ indistintamente com e sem filtro. Dentre as mu1heres, 2,6\% fumavam exclusivamente pito de barro (todas com idade de 55 anos e mais), hábito também típico de zona rural; $0,7 \%$ fumava exclusivamente cigarros de palha, e $96,7 \%$ fumavam exclusivamente cigarros de papel com filtro. 
Tabela 3. Prevalência (\%) de tabagismo em número de cigarros fumados por dia (ou equivalência) e idade, população de 15 a 74 anos do sexo masculino. Município de Araraquara, SP, zona urbana, 1987.

\begin{tabular}{lrrrrrrr}
\hline $\begin{array}{c}7 \\
\text { N } \text { de cigarros } \\
\text { fumados por dia ou } \\
\text { equivaléncia }\end{array}$ & $15-24$ & $25-34$ & $35-44$ & $45-54$ & $55-64$ & $65-74$ & Total \\
\cline { 2 - 8 } & & & & & & & \\
Não fumantes & 67,1 & 46,8 & 44,5 & 63,0 & 55,5 & 42,0 & 54,8 \\
ou ex-fumantes & 5,7 & 6,3 & 4,9 & 5,5 & 13,8 & 16,1 & 7,3 \\
$1-9$ & 14,3 & 13,3 & 2,5 & 13,7 & 7,7 & 12,9 & 11,3 \\
$10-19$ & 12,9 & 29,4 & 33,3 & 13,7 & 21,5 & 29,0 & 22,5 \\
$20-39$ & - & 4,2 & 14,8 & 4,1 & 1,5 & - & 4,1 \\
$\geq 40$ & 100,0 & 100,0 & 100,0 & 100,0 & 100,0 & 100,0 & 100,0 \\
\hline Total & 100,0 &
\end{tabular}

Tabela 4. Prevalência (\%) de tabagismo em número de cigarros fumados por dia (ou equivalência) e idade, população de 15 a 74 anos do sexo feminino. Município de Araraquara, SP, zona urbana, 1987.

\begin{tabular}{lrrrrrrr}
\hline $\begin{array}{l}\text { N2 de cigarros } \\
\text { fumados por dia ou } \\
\text { equivalência }\end{array}$ & $15-24$ & $25-34$ & $35-44$ & $45-54$ & $55-64$ & $65-74$ & Total \\
\cline { 2 - 8 } & & & & & & & \\
Não fumantes & 77,1 & 62,8 & 81,4 & 82,8 & 83,5 & 93,4 & 77,2 \\
ou ex-fumantes & 14,0 & 14,3 & 5,3 & 8,6 & 10,6 & 2,2 & 10,5 \\
$1-9$ & 4,5 & 11,8 & 7,1 & 3,8 & 5,9 & 2,2 & 6,6 \\
$10-19$ & 3,8 & 9,9 & 5,3 & 3,8 & - & 2,2 & 5,0 \\
$20-39$ & 0,6 & 1,2 & 0,9 & 1,0 & - & - & 0,8 \\
$\geq 40$ & 100,0 & 100,0 & 100,0 & 100,0 & 100,0 & 100,0 & 100,0 \\
\hline Total & & & & & & &
\end{tabular}

Dentre os homens que fumavam cigarros de papel, apenas $17,8 \%$ afirmavam usar cigarros de baixo teor de nicotina. Nas mulheres, esta proporção se elevava para $42,1 \%$.

Os tabagistas do sexo masculino referiam nunca tragar em $6,7 \%$ dos casos; $11,6 \%$ às vezes tragavam; e $81,7 \%$ sempre tragavam. As mutheres tabagistas referiam nunca tragar em $11,8 \%$ dos casos; $14,5 \%$ às vezes tragavam; e $73,7 \%$ tragavam sempre.

A Tabela 5 apresenta a prevalência de tabagismo segundo sexo e estratos de renda familiar. Nota-se um nítido predomínio em ambos os sexos do hábito de fumar, no estrato de renda familiar mais baixa.

Tabela 5. Prevalência (\%) de tabagismo na população urbana de 15 a 74 anos segundo os estratos de renda familiar* e sexo. Município de Araraquara, SP, zona urbana, 1987.

\begin{tabular}{lccc}
\hline Estratos & \multicolumn{2}{c}{ Sexo } & \\
\cline { 2 - 3 } Re & Masculino & Feminino & Total \\
\hline Baixa (<5,0 PNS) & 52,0 & 25,4 & 36,7 \\
Média ( 5 - 9,0 PNS) & 46,6 & 24,5 & 34,5 \\
Alta ( 210,0 PNS) & 41,2 & 19,3 & 28,8 \\
\hline Total & 45,2 & 22,8 & 32,8 \\
\hline
\end{tabular}

* Exclusive renda ignorada.

PNS: Pisos Nacionais de Salátio.
Tabela 6. Prevalência (\%) de tabagismo na população de 15 a 74 anos, segundo escolaridade e sexo. Município de Araraquara. SP, zona urbana, 1987.

\begin{tabular}{lccc}
\hline $\begin{array}{l}\text { Grau de } \\
\text { Escolaridade } \\
\text { (anos de escolaridade) }\end{array}$ & Masculino & Feminino & Total \\
\cline { 2 - 3 } & \multicolumn{2}{c}{ Sexo } \\
\hline $\begin{array}{l}\text { Analfabeto a < } 4 \text { anos } \\
\text { (primário incompleto) }\end{array}$ & 48,6 & 21,5 & 32,3 \\
$\begin{array}{l}4-7 \\
\text { (primário completo) }\end{array}$ & 48,8 & 26,5 & 36,5 \\
$\begin{array}{l}8-10 \\
\text { (ginásio completo) }\end{array}$ & 47,8 & 22,7 & 32,9 \\
$\begin{array}{l}11-13 \\
\text { (colegial completo) }\end{array}$ & 38,1 & 20,2 & 29,8 \\
$\begin{array}{l}\geq 14 \\
\text { (universidade completa) }\end{array}$ & 35,7 & 21,2 & 32,8 \\
\hline Total & 45,2 & 22,8 & 32,8 \\
\hline
\end{tabular}

A Tabela 6 mostra a prevalência de tabagismo segundo sexo e escolaridade formal. Nota-se que quanto maior o grau de escolaridade entre os homens, menor a prevalência de tabagismo. Já entre as mulheres, estas diferenças não são tão nítidas.

\section{Discussáo}

Os resultados apontam para uma alta prevalên- 
cia do hábito de fumar, predominando em homens mais velhos e de renda e escolaridade mais baixas. Contudo, no grupo etário mais jovem já existe uma alta proporção de pessoas que se iniciaram no tabagismo. Sabe-se que os esforços internacionais têm se concentrado na prevenção do tabagismo na adolescência.

A proporção de ex-fumantes é maior entre os homens, possivelmente em decorrência de serem eles os que mais fumam. Os homens não apenas fumam mais frequientemente do que as mulheres, como fumam em maior quantidade cigarros de mais alto teor de nicotina, e tragam mais frequientemente.

Os resultados são compativeis com os dados da literatura ${ }^{1,2} \mathrm{com}$ referência às variáveis sóciodemográficas. Entretanto, pode-se verificar que a magnitude da prevalência do tabagismo é bem mais alta em Araraquara do que aquela observada em alguns países das Américas ${ }^{1}$.

Por outro lado, inquérito realizado na própria localidade estudada mostrou uma prevalência de $6,7 \%$ de tabagismo entre escolares de primeiro e segundo graus, tendo a maioria iniciado o hábito entre 12 e 17 anos, com uma ligeira preponderância das mulheres sobre os homens ${ }^{6}$.

Pode-se verificar assim que a população urbana do município, que representa o interior afluente urbano do Estado de São Paulo, está exposta a pelo menos três importantes fatores de risco para doenças crônicas não-transmissiveis: hipertensão arterial $^{3,5}$, obesidade ${ }^{4}$ e tabagismo.

Tudo isto torna o combate e o controle dessas entidades prioritário para qualquer programa de prevenção das doenças crônicas em cidades semelhantes do Estado de São Paulo e do Brasil.

LOLIO, C. A. de et al. [Prevalence of smoking in a city of southeastern Brazil]. Rev. Saúde Pública, 27: $262-5,1993$. A cross-sectional study for prevalence of arterial hipertension in the population aged 15.74 years of age of the urban area of Araraquara County, $250 \mathrm{~km}$ from the city of S. Paulo, S. Paulo, State,
Brazil, in 1987, was performed. The questionnaires presented to 1,199 people (533 men and 666 women) at the interview consisted of regarding sociodemographic variables, as well as the use of tobacco (smoking), the ways in which tobacco was used and the habit of inhaling the smoke. The sample was taken by the procedure of clustering, carried out in three stages. The sample was equiprobabilistic. The prevalence of tobacco smoking was very high being of $45.2 \%$ for men and $22.8 \%$ for women. The exsmokers accounted for $15.9 \%$ of men and $8.0 \%$ of women. The men somked much more than the women. The poorer smoked more than the richer, in both sexes. Among men, the prevalence of smoking was inversely proportional to duration of schooling, but that difference was not noted in women. As there have been other studies regarding the high prevalence of obesity and high blood pressure, it was conluded therefore that the population of Araraquara, an average town of the affluent urban interior of S. Paulo State, has a high frequency of risk factors for chronic non-transmissible diseases.

Keywords: Smoking, epidemiology. Risk factors. Chronic disease, epidemiology.

\section{Referências Bibliográficas}

1. El TABAQUISMO en las Américas . Bol. Epidem OPS, 10 (3): 1-5, 1989.

2. FIORE, M.C. et al. Cigarette smoking: the clinican's role in cessation, prevention and public health. Dis. Mon., 35: 183-205, 1990.

3. LOLIO, C.A. de. Prevalência da hipertensão arterial em Araraquara. Arq. Bras. Cardiol., 55: 167-73, 1990.

4. LOLIO, C.A. de \& LATORRE, M. do R. D.O. Prevalência da obesidade em localidade do Estado de São Paulo, Brasil, 1987. Rev. Saúde Pública, 25: 33-7, 1991.

5. LOLIO, C.A. de. Prevalência de hipertensão arterial no Município de Araraquara, SP, em 1987. São Paulo, 1989. [Tese de Doutorado - Faculdade de Medicina da USP].

6. SIMÓES, M.J.S. Tabagismo entre estudantes do primeiro e segundo graus em Araraquara, SP, Brasil, 1988. Medicina, Ribeirão Preto, 23: 223-31, 1990.

Recebido para publicação em 21.12.1992 Aprovado para publicação em 5.7.1993 